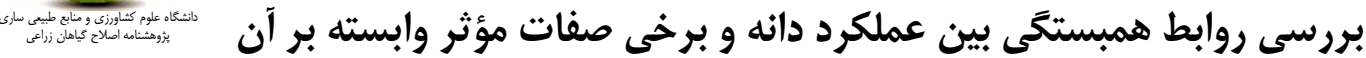

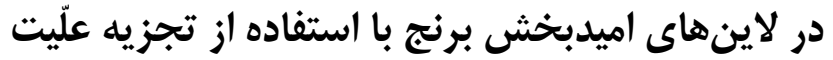

\author{
مريم سرائى'، مر تضى سامدليرى '، على محدثى 'و مجيد مرادخانى
}

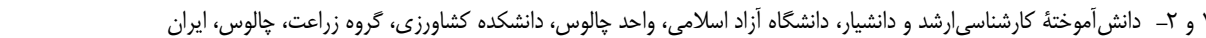

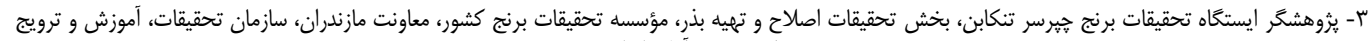

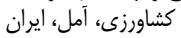
عا- دانش آموختهُ كارشناسىارشد، دانشكاه آزاد اسلامى، واحد تاكستان، دانشكده علوم كشاورزى، كروه زراعت، تاكستان، ايران

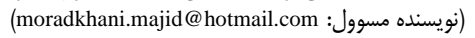

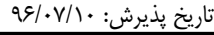
تاريخ دريافت:

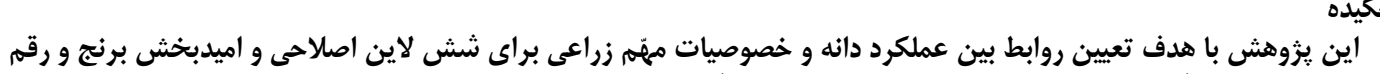

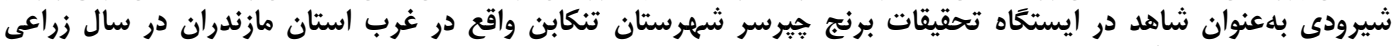

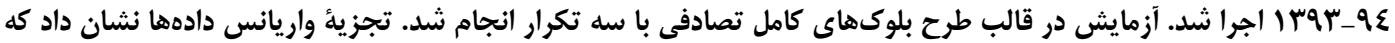

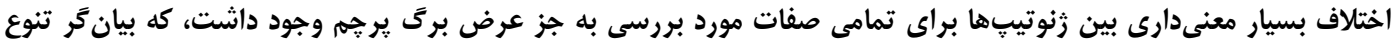

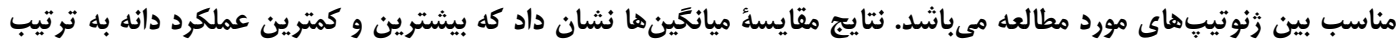

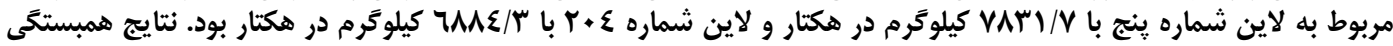

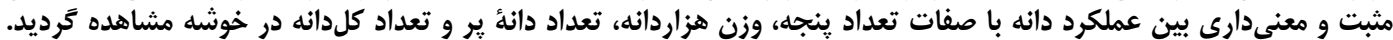

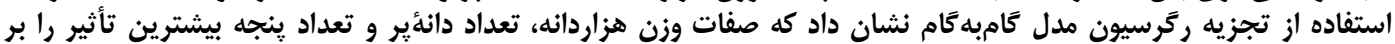

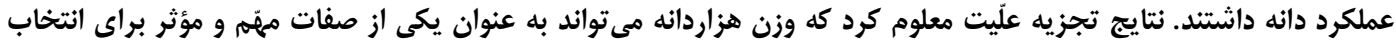

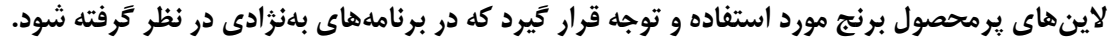

وازههاى كليدى: ارقام برنج، تجزيه رَّرسيون مدل كامبه كام، تجزيه مسير، تجزيه همبستكى صفات، مقايسهُ عملكرد

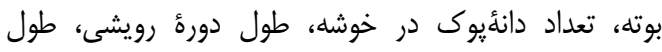

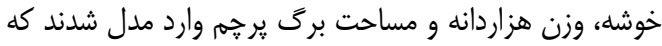

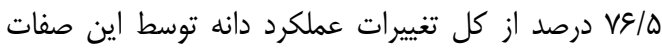

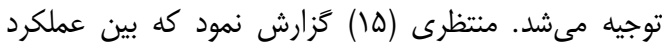

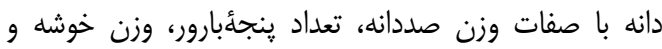

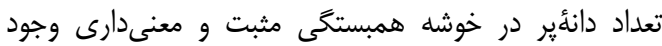

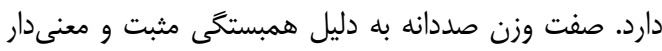

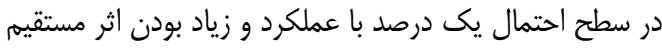

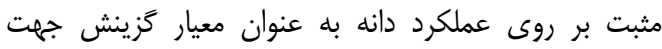

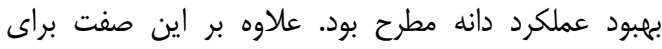

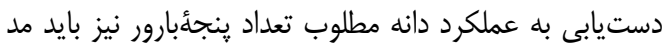

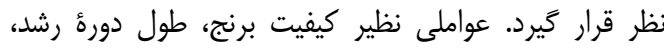

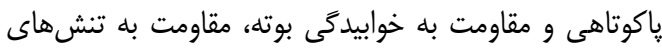

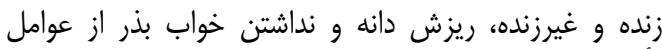

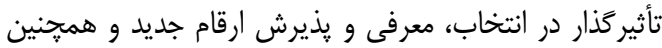

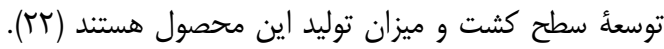

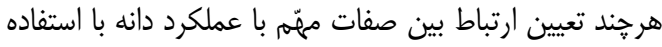

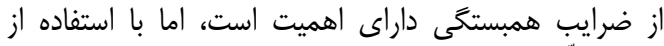

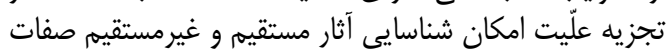

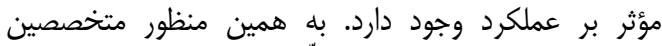

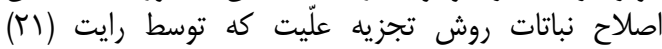

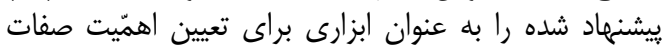

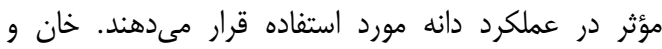

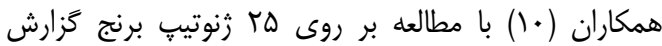

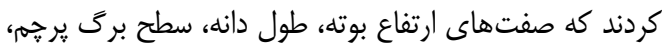

كشور ما تاكنون از نظر توليد برنج (Oryza sativa L.)

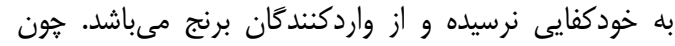

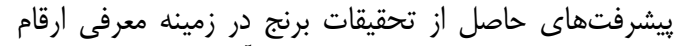

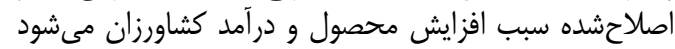

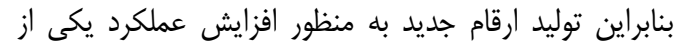

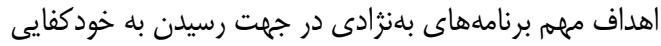

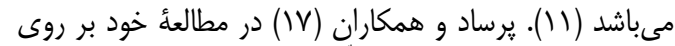
همبستخى صفات و تجزئئ علّيت در برنج

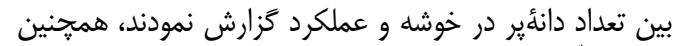

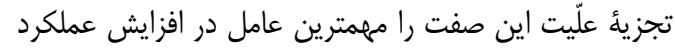

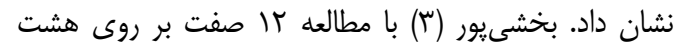

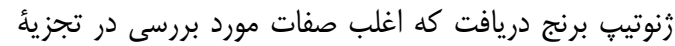

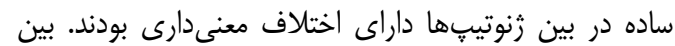

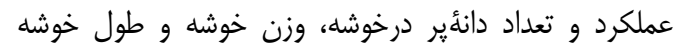

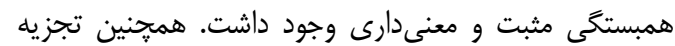

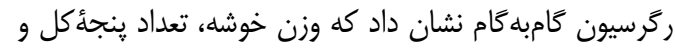

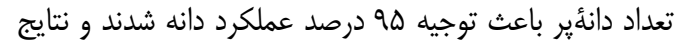

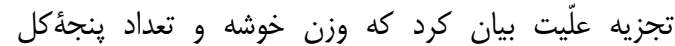

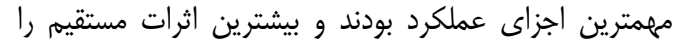

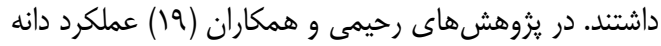

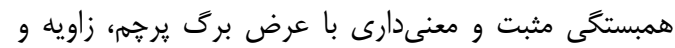

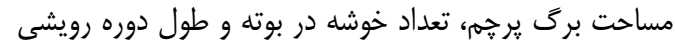

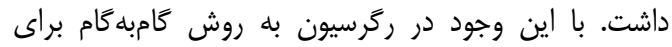

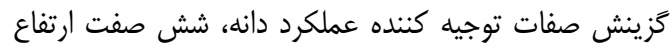


عملكرد و مطالعأ اثرات مستقيم و غيرمستقيم اين اجزا با داب

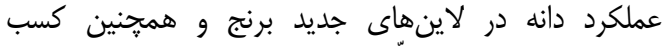

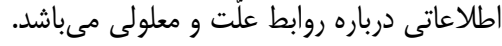

\section{مواد و روشها}

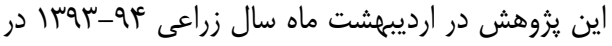

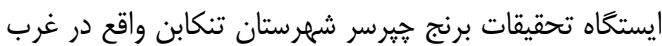

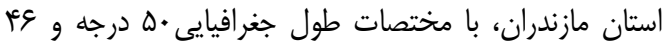

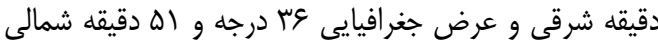

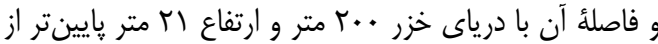

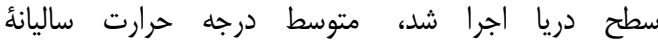

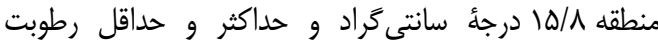

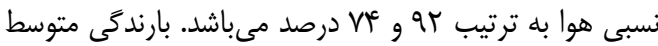

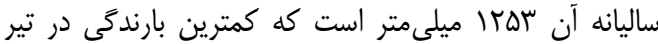

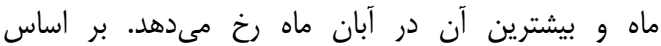

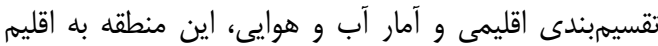

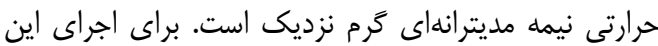

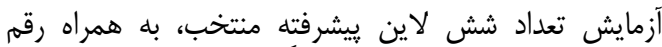

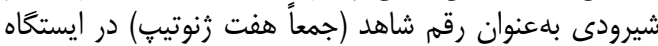

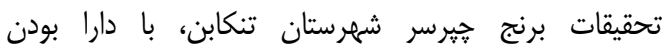

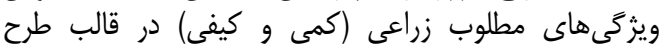

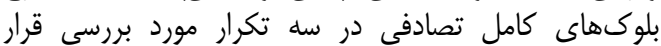

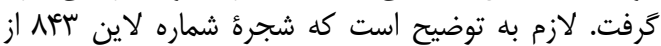

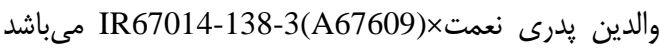

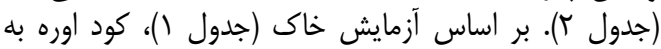

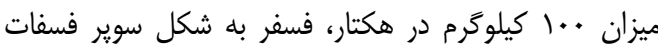

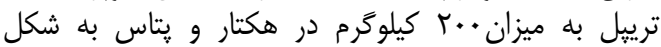

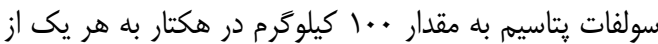

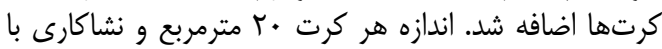

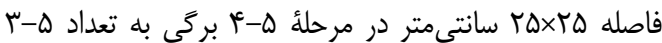

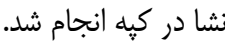

تعداد دانه در خوشه و تعداد ينجه در گئياه با عملكرد رابطه

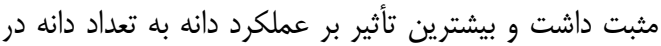

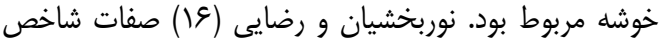

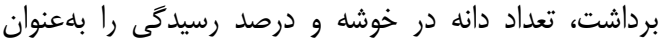

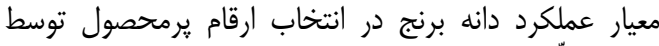

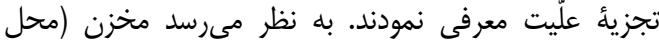

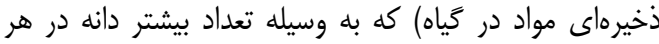

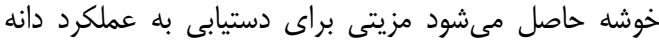

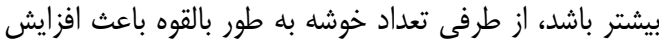

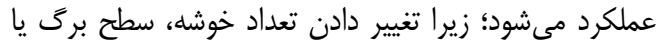

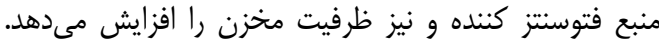

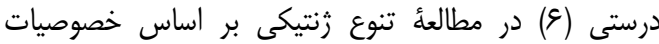

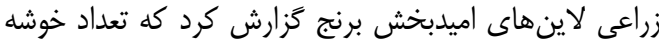

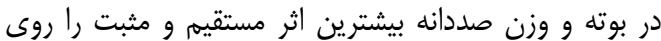

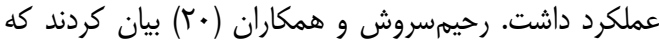

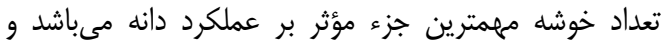

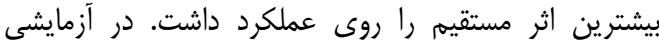

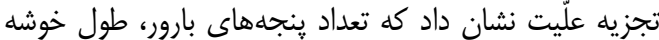

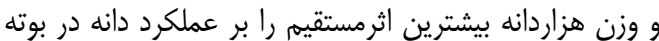

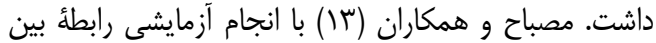

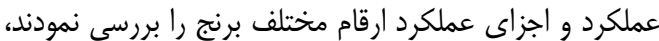

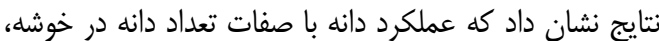

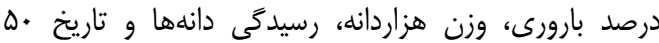

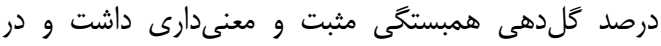

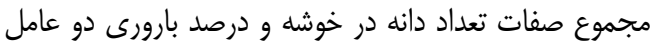

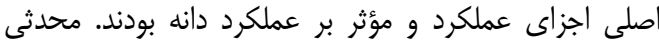

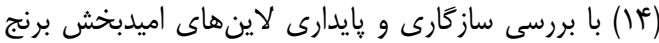

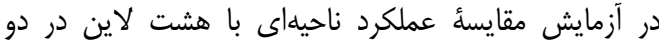

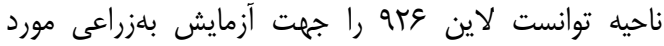

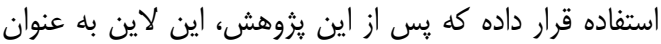

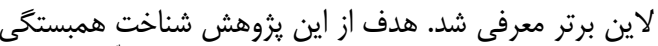

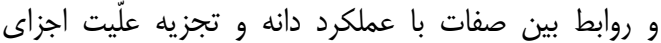

Table 1. Physical and chemical characteristics of soil before planting of rice

جدول 1- خصوصيات فيزيكى و شيميايى خاك قبل از نشاكارى برنج

\begin{tabular}{|c|c|c|c|c|c|c|c|c|c|c|}
\hline $\begin{array}{c}\text { ش } \\
\text { Sand }\end{array}$ & 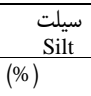 & $\begin{array}{c}\text { ر) } \\
\text { Clay }\end{array}$ & قابل جذب $\begin{array}{c}\text { قئاس } \\
\text { (ppm) }\end{array}$ & $\begin{array}{c}\text { قابل جذب } \\
\text { (ppm) }\end{array}$ & 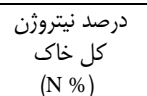 & $\begin{array}{c}\text { درصد كربن } \\
\text { آلى (O.C \%) }\end{array}$ & $\begin{array}{c}\text { خنثى شونده } 2 \text { مواد } \\
\text { (TNV) }\end{array}$ & $\begin{array}{c}\text { اسيديته } \\
\text { pH }\end{array}$ & 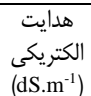 & $\begin{array}{l}\text { خاك } \\
\text { (cm) }\end{array}$ \\
\hline$\Delta / V$ & $\mathrm{ra} / \mathrm{M}$ & $\Delta Q / \vee q$ & $1 \%$. & $r \in / T$ &.$/ T K$ & $T / \Delta V$ & $r / T)$ & $8 / 91$ & . & \\
\hline
\end{tabular}

Table 2. Characteristics of lines and varieties used in the study

\begin{tabular}{|c|c|c|}
\hline شماره لاين & والدين يدرى" & والدين مادرى \\
\hline لاين I & شماره • ا از رقم شيرودى & ديلمانى \\
\hline لاين & شماره • ا از رقم شيرودى & R67015/22/6/2/(A67602) of number 59 شمارهץ × (آملץ) \\
\hline لاين & 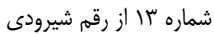 & R67015/22/6/2/(A67602) of number 59 شمارهץ × (آملَ) \\
\hline لاين F الما & شماره ا از سع人 & R67015/22/6/2/(A67602) of number 111 شمارهب × (آمل س) \\
\hline لاين ه ا & شماره r از رقم ديلمانى & $(111 \times$ IR 192$)$ of number 10 \\
\hline 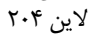 & 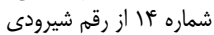 & (IR67015/22/6/2(A37632) × شمارهץ × (آمل؟) \\
\hline 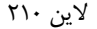 & رقم شيرودى - ن م & شاهد \\
\hline
\end{tabular}


محصول بين آغازش خوشه و گردهافشانى است و اين صفت

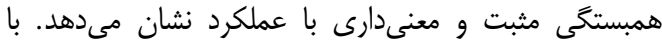

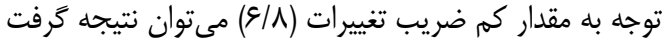

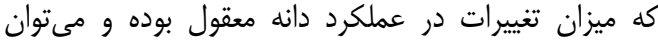

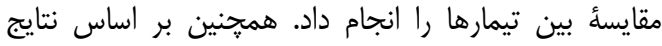

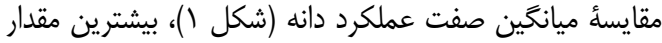

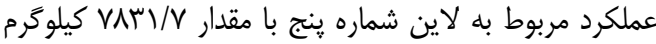

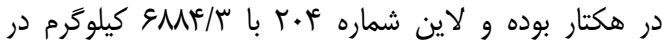

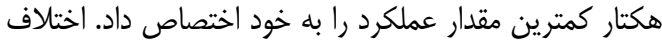

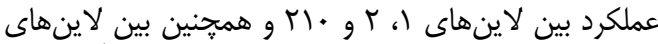

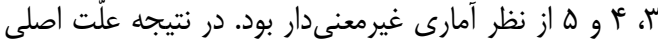

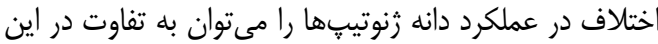

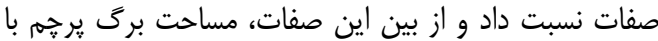

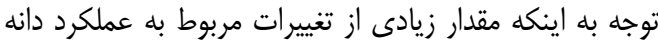

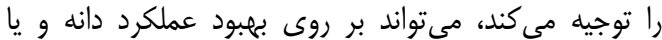

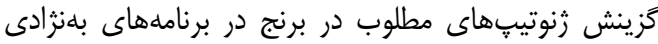

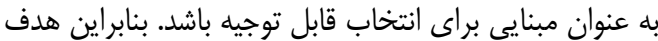

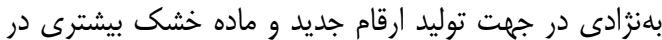

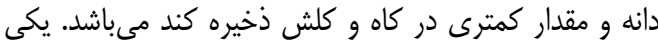

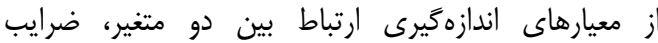

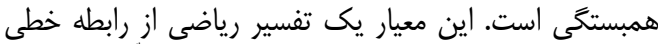

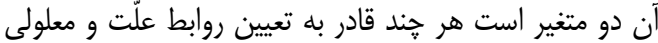

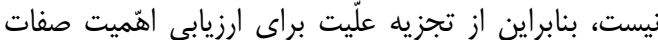

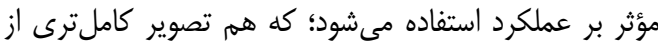

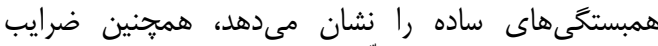

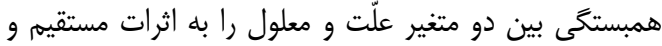

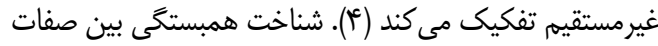

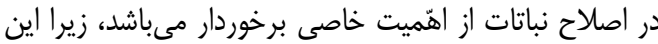

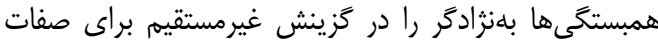

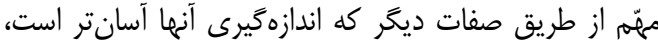

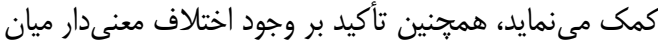

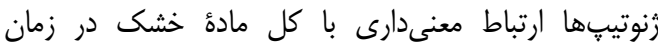

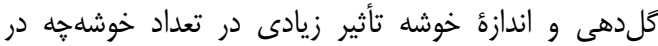

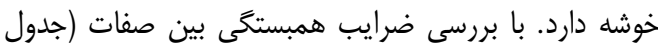

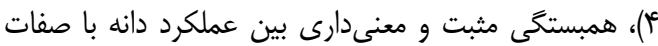

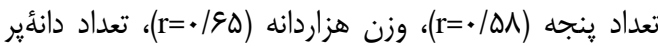

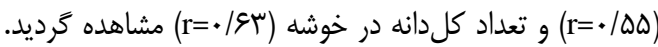

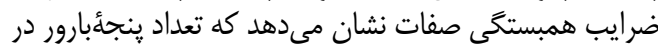

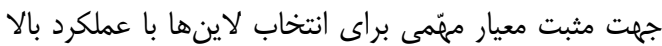

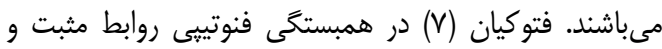

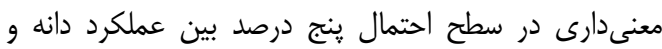

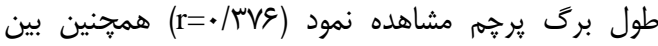

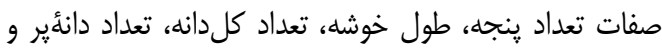

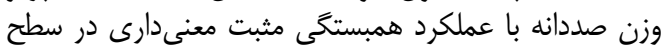
احتمال يك درصد گزارش نمود

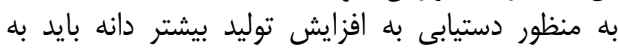

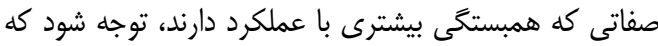

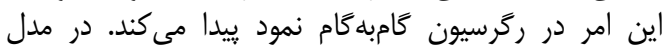

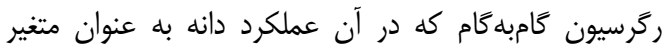

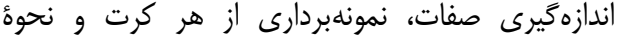
محاسبة صفات بر اساس روش استاندارد ارزيابى درئ در مؤسسه

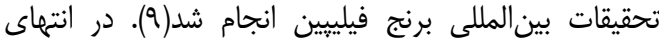

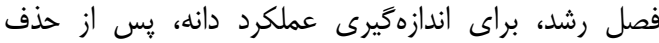

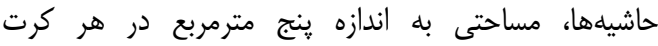

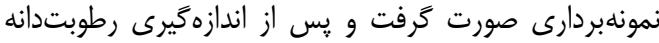

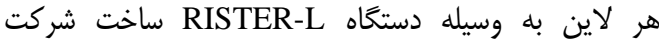
KIYA SEISAKUSHO

M = ( كه در آن M وزن محصول در رطوبت استاندارد (fأدرصد)،

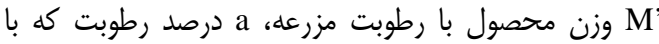

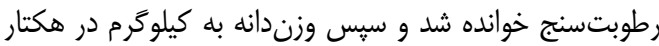

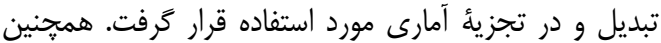

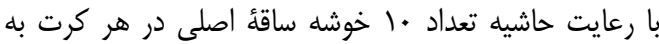

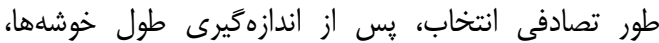

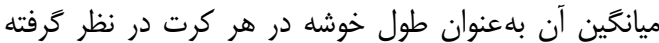

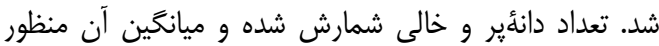

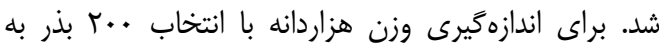

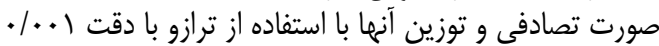
كرم صورت گرفت.

تجزيه و تحليلهاى آمارى دادهها شامل شارل آزمون نرمال

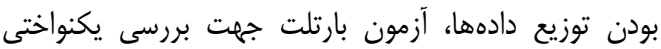

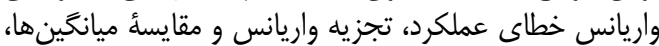

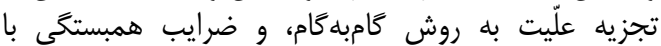

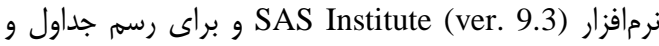

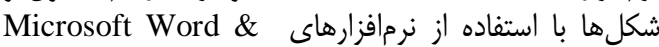

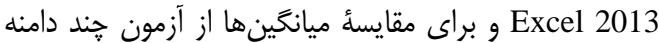
اى دانكن در سطح احتمال ينج درصد استفاده شد.

\section{نتايج و بحث}

بر اساس نتايج تجزيه واريانس (جدول بـ بـان، بين لاينهاى

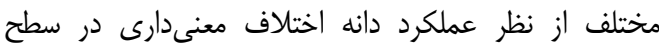

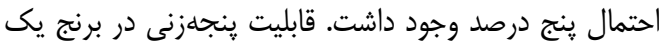

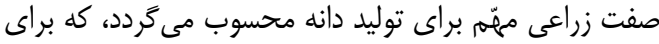

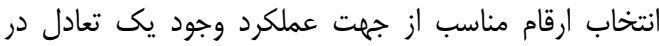

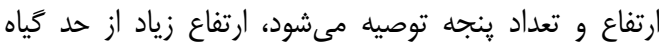

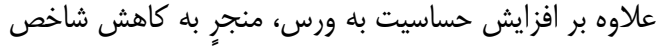

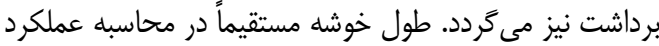

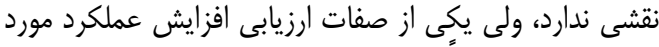

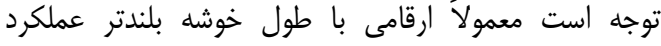

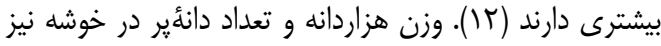

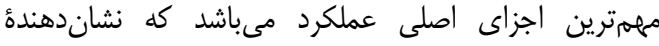

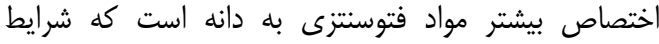

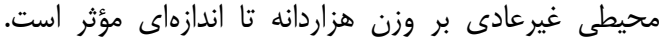

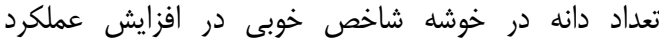

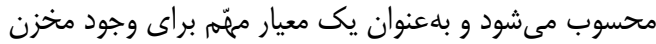

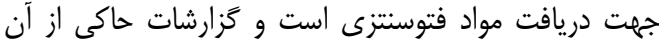

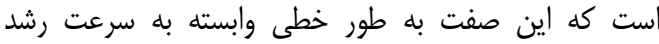


تعداد ينجأبارور، رحيمسروش و همكاران (•r) تعداد خوشه در

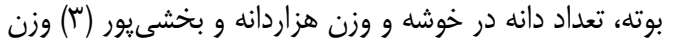

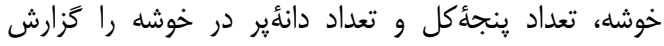

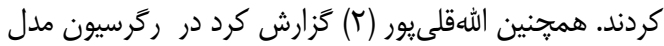

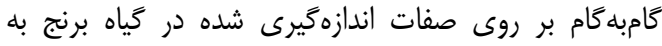

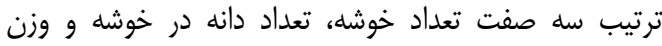

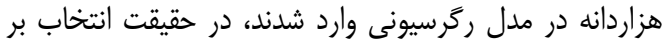

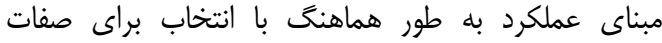

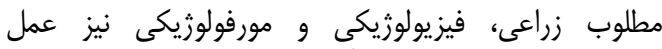

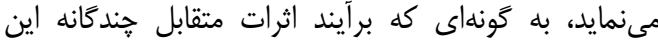

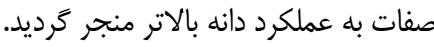

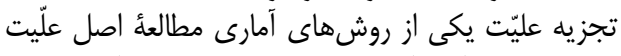

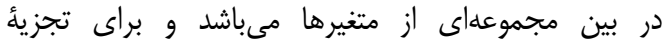

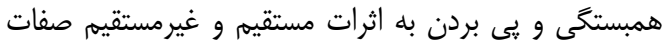
بسيار مفيد است (ه). در اين بروهش اثر اثرمستقيم و غيرمستقيميم

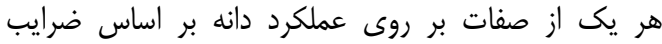

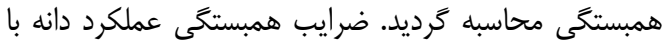

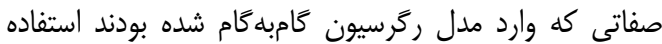

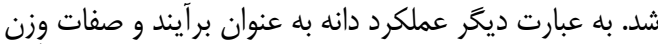

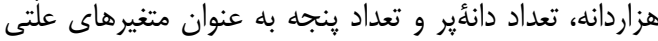

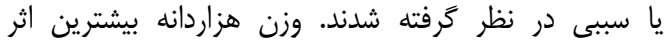

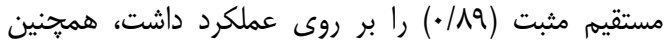

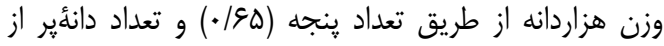

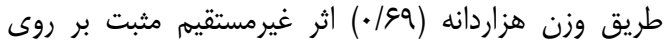

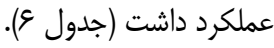

وابسته (معلول) در برابر صفات ديخر به عنوان متغيرهاى

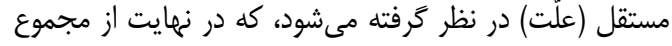

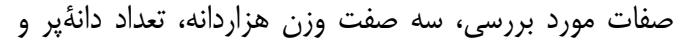

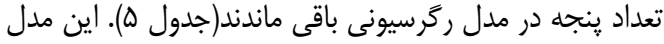

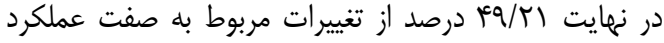

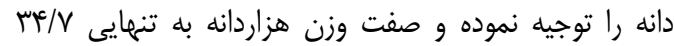

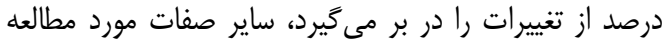

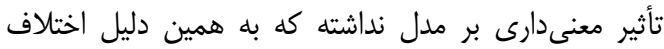

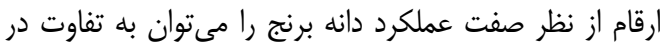

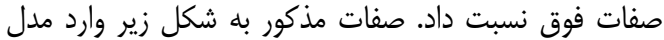

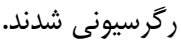

$\mathrm{Y}=1092.7+44.73 \mathrm{x}_{8}+42.51 \mathrm{x}_{9}+37.46 \mathrm{x}_{2}$

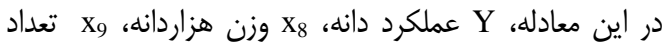

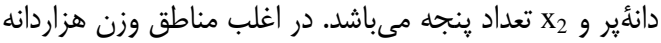

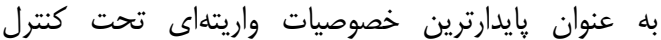

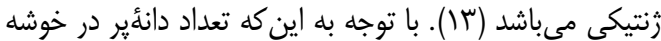

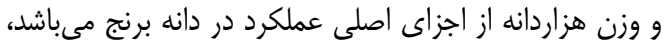

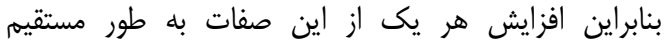

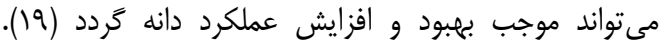

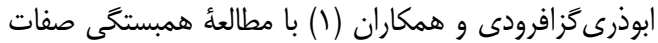

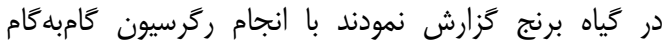

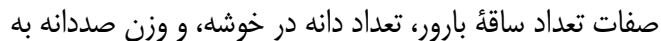

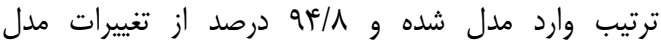

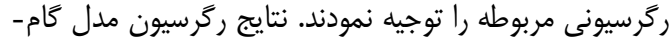

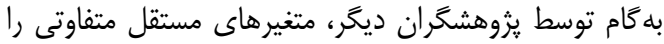

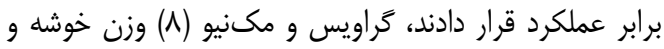

Table 3. Analysis of variance (mean squares) for studied traits in rice genotypes

\begin{tabular}{|c|c|c|c|c|c|c|c|c|c|c|c|c|c|c|}
\hline تغييرات & آزادى درجه & عملكرد دانه & رطوبت & كل تعداده & دانهخالى & دانعداد & هزاردانه & عرضه & طول دانه & خوشه & 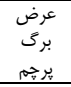 & برّى طولم & تعنجه & ارتفاع بوته \\
\hline تكرار & r & DQFGST & $1 / M r^{\mathrm{ns}}$ & $V N / V r^{\text {ns }}$ & $1 \cdot 1 / 8^{\mathrm{ns}}$ & $r \cdot / 9 q^{\mathrm{ns}}$ & $r / \Lambda^{\mathrm{ns}}$ & $\cdot / \cdots v^{\mathrm{ns}}$ & $\cdot / \ldots c^{\mathrm{ns}}$ & $\cdot / 8^{\mathrm{ns}}$ & $\cdot / \cdot 1^{\mathrm{ns}}$ & $N / \cdot \Delta^{\mathrm{ns}}$ & $\varphi / \Gamma \omega^{\mathrm{ns}}$ & $10 / q^{n s}$ \\
\hline خطيمار & 9 & TForrT/qf* & $1 / \cdot v^{* * *}$ & $1 \pi \cdot 9 / r^{* * *}$ & $V \backslash \Delta / q^{* *}$ & $1995 / 99^{*}$ & $\mid r / r \varepsilon^{* * *}$ & $\cdot / \cdot r^{* * *}$ & $\cdot / \cdot v^{* * *}$ & $\cdot / \cdot v^{*}$ & $\because / *^{\mathrm{ns}}$ & $r r / q F^{*}$ & $r \cdot / v \varepsilon^{*}$ & 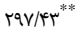 \\
\hline آزمايش حطاى & ir & TQFA. $9 / F^{\leftarrow}$ & $\cdot / \pi$ & $\mid T V / A f$ & $\mid T V / A r$ & $1 \% 8 / 99$ & $\Delta / .9$ & 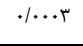 &.$/ . .1$ & $\cdot / \Delta$ & $\%$ & $11 / 9$ & $N / r$ & $r \Delta / N T$ \\
\hline $\begin{array}{l}\text { C.V } \\
(\%)\end{array}$ & - & $8 / 1$ & $\Gamma / \Lambda$ & $V / q$ & $r q / 4$ & $9 / M$ & $\mathrm{~N} \cdot \mathrm{V}$ & $N / T$ & $T / \mu r$ & $T / \Delta$ & $1 \cdot / 4$ & $N / 8$ & $I T / \Delta$ & $F / \Delta$ \\
\hline
\end{tabular}


جدول عا ضرايب همبستخى ييرسون بين صفات مورد مطالعه در لاينهاى برنج

Table 4. Correlation coefficient pearson between traits studied in rice lines

\begin{tabular}{|c|c|c|c|c|c|c|c|c|c|c|c|c|c|}
\hline & 1 & $r$ & $r$ & f & $\Delta$ & 8 & $\mathrm{~V}$ & $\Lambda$ & 9 & 1. & 11 & IT & Ir \\
\hline ارتفاع بوته -1 ا & 1 & & & & & & & & & & & & \\
\hline تعداد ينجه در بوته -r & איז/. & 1 & & & & & & & & & & & \\
\hline طول برى يرجم - &.$/ 9 V^{* \pi}$ &.$/ \cdot r$ & 1 & & & & & & & & & & \\
\hline عرض برك يرحم - f & $\cdot \mid a Y^{n+*}$ &.$/ 11$ &.$/ 19^{* *}$ & 1 & & & & & & & & & \\
\hline طول خوشه -ه & 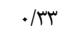 &.$/ T V$ & $\cdot / \Delta \Delta^{\prime \prime}$ & $\cdot / \Delta T^{\circ}$ & 1 & & & & & & & & \\
\hline طول دانه -ع & $-\cdot / \mu$ &.$/ 1 f$ & $-+/ \cdot V$ & ./.r &.$- / 19$ & 1 & & & & & & & \\
\hline ع- عرض دانه & $-\cdot / 4 V^{\circ}$ & $-\cdot / 1 \mathrm{~V}$ & $.1 \cdot 1$ & $\cdot / 1$ &.$/ 1 \mathrm{~V}$ & 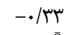 & 1 & & & & & & \\
\hline ئن هزار دانه -1 & .1 .9 & r & $-\cdot / r r$ & $-\cdot / \cdot 1$ & $-\cdot / r V$ &.$/ 48^{*}$ & $\cdot / T F$ & 1 & & & & & \\
\hline 9عداد دانهير -9 &.$/ \Delta T^{\circ}$ & $-\cdot / r$ & $.1 \cdot 0$ &.$/ 19$ &.$/ 19$ & $\cdot / 4 \Delta^{\circ}$ & $\cdot / \Delta \varphi^{N+*}$ &.$/ \cdot r$ & 1 & & & & \\
\hline تعداد دانه يوى --1. &.$/ 11$ & -.1 .9 & $\cdot / \kappa^{\circ}$ & 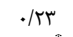 & .1 .0 &.$- / 19$ &.$- / \cdot \varphi^{c}$ & ع ع/.- - ( & $-\cdot / 19$ & 1 & & & \\
\hline تعداد كل دانه -11 & $.18^{-n}$ &.$- / \cdot \Delta$ &.$/ 4 \Lambda^{\circ}$ &.$/ 4 q^{*}$ & $\cdot / \Delta f^{\circ}$ & $-* / 4 f^{*}$ & $\cdot / 4 q$ & عس/•- & $\cdot / 99^{N+*}$ & $\cdot / r v$ & 1 & & \\
\hline درصد رطوبت دانه - IT & . &.$- / 19$ & $-+/ \cdot V$ &.$- / 19$ & $-\cdot / r$ & $-\cdot / 14$ & $\cdot / 1$ &.$/ .4$ & $-\cdot / \cdot r$ & $\cdot / F F^{\prime}$ &.$/ 1 r$ & 1 & \\
\hline عملكرد دانه-"r| & $\cdot / \cdot V$ & $\cdot / \Delta \Lambda$ & $\cdot / T \Delta$ & $\cdot / K F$ & .110 &.$/ \cdot r$ & كس/. &.$/ 9 \Delta^{* *}$ & $\cdot / \Delta \Delta^{*}$ & $.1 \cdot 9$ & $\cdot \mid \& r^{* * *}$ & $\cdot / \cdot r$ & 1 \\
\hline
\end{tabular}

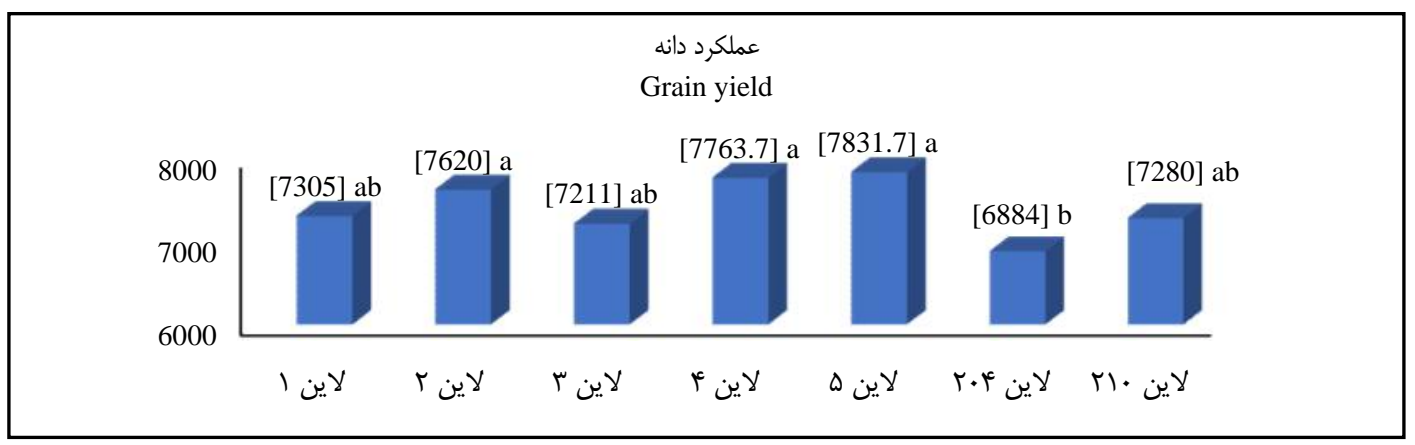

شكل ا- مقايسه ميانكين عملكرد دانهُ زنوتيبٍ هاى مختلف برنج

Figure 1. Mean comparison of grain yield in rice different genotypes

جدول ه- ركرسيون مدل كامبه كام براى عملكرد دانه بهعنوان متغير وابسته و ساير صفات بلهنوان متغير مستقل Table 5. Stepwise regression method for grain yield as dependent variable and other traits as independent variables

\begin{tabular}{|c|c|c|c|}
\hline \multicolumn{4}{|c|}{ مراحل رگرسيون كامبهُام } \\
\hline صفات در مدل & 1 & $r$ & $r$ \\
\hline عرض از مبدأ & ITVAS & IrTqF/T & $1.9 Y / V$ \\
\hline وزن هزاردانه & $+\Delta 9 / \Delta 1$ & $+f V / f \Delta$ & $+F F / V^{* *}$ \\
\hline تعداد دانهُير & & $r \Delta / T^{-}$ & $F Y /\left.D\right|^{* *}$ \\
\hline تعداد ينجه در بوته & & & $r V / \& \varepsilon^{*}$ \\
\hline ضريب تبيين & $M F / V$ & ואו & $89 / 71$ \\
\hline
\end{tabular}

جدول ك- تجزيه علّيت همبستخى عملكرد دانه با صفات باقىمانده در مدل ركرسيونى كامبه كام

Table 6. Path analysis between grain yield and other remaining variables in stepwise regression method

\begin{tabular}{|c|c|c|c|c|c|}
\hline \multirow{2}{*}{ اثر كل } & \multicolumn{3}{|c|}{ اثرات غيرمستقيم } & \multirow{2}{*}{ اثرمستقيه } & \multirow{2}{*}{ صفات } \\
\hline & تعداد ينجه در بوته & تعلداد دانٔئير & وزن هزاردانه & & \\
\hline$\cdot|\Delta|$ & 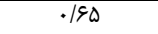 & . & ----- &.$/ 19$ & وزن هزاردانه \\
\hline • $/ 4 F$ & $\cdot / \Delta \Delta$ & ----- & .199 & $\cdot / 4$ & تعداد دانٔهير \\
\hline.$/ \mathrm{V}$ & ----- & $-\cdot / \pi$ & .19 & $\cdot / 1$ & تعداد ينجه در بوته \\
\hline & & & & . & اثر باقىمانده \\
\hline
\end{tabular}




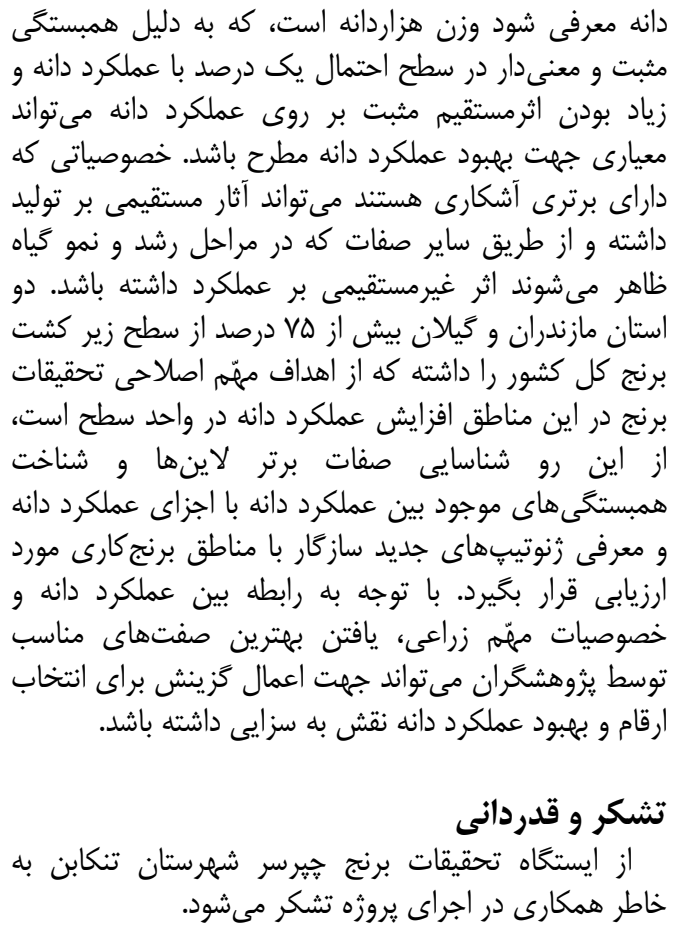

تجزيه واريانس نشان داد كه اختلاف بسيار معنىدارى

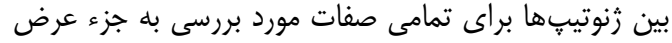

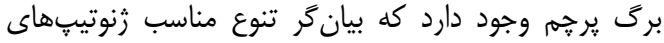

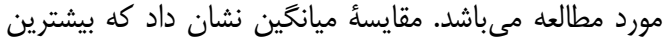

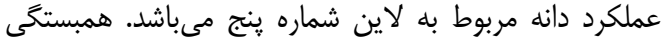

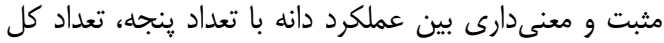

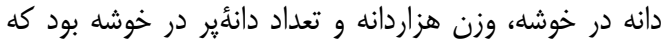

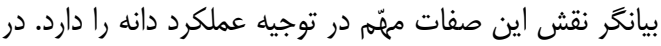

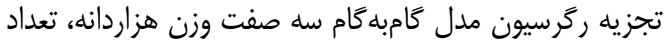

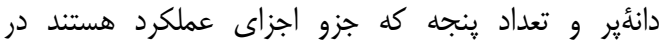

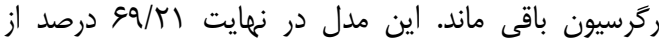

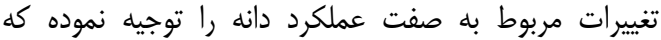

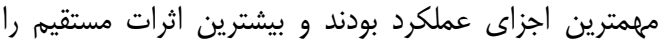

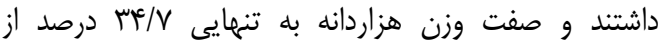

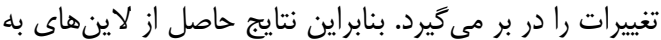

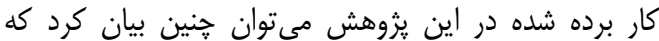

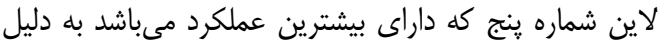

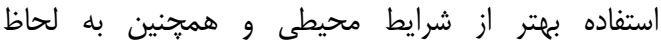

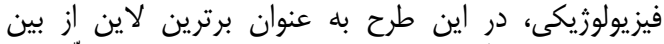

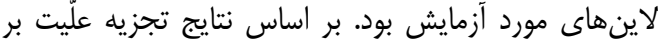

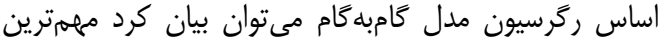

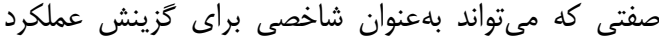

1. Abozari-gezafrodi, A., R. Honar-nejad, M.H. Fotokian and A. Alami. 2006. Agronomic traits correlation study and path analysis in rice. Science and Technology of Agriculture and Natural Resources, 10(2): 99-106 (In Persian).

2. Alah-gholipour, M. 1997. Investigation of correlation between some important agronomic traits with a yield of path analysis in rice. M.Sc. Thesis, Tehran University, Faculty of Agriculture and Natural Resources, Karaj, Iran, 105 pp (In Persian).

3. Bakhshi-pour, S. 2006. Study of morphological characteristics and yield components of rice lines in two regions of the Mazandaran province. M.Sc. Thesis, Islamic Azad University, Bojnourd Branch, Faculty of Agriculture, Bojnourd, Iran, $135 \mathrm{pp}$ (In Persian).

4. Balouchzaehi, A. and G. Kiani. 2013. Determination of selection criteria for yield improvement in rice. Journal of Crop Breeding, 5: 75-84 (In Persian).

5. Dewey, D.R. and K.M. Lu. 1959. A correlation and path coefficient analysis of components of crested wheat grass seed production. Agronomy Journal, 51: 197-203.

6. Dorosti, H. 2000. Genetic variation based on agronomic characteristics of rice lines. M.Sc. thesis, Islamic Azad University, Karaj Branch, Karaj, Iran, 79 pp (In Persian).

7. Fotokian, H. 2014. Study of agronomic traits and yield of rice lines in west mazandaran. M.Sc. thesis, Islamic Azad University, Chalous Branch, Faculty of Agriculture, Agronomy Department, Chalous, Iran, 73 pp (In Persian).

8. Gravios, K.A. and R.W. Mcnew. 1993. Genetic relationship among and selection for rice yield and yield component. Crop Science, 33: 249-259.

9. IRRI. 2013. Standard evaluation system for rice $5^{\text {th }}$ edition (SES). International Rice Research Institute, Manila, Philippines.

10. Khan, A.S., M. Irman and M. Ashfaq. 2009. Estimation of genetic variability and correlation for grain yield components in rice. American Eurasian Journal Agriculture and Environ Science, 6(5): 585-590.

11. Mahabub, H. 2005. Does rice research reduce poverty in asia? Rice Today, 5(1): $37 \mathrm{pp}$.

12. Mehter, S.S., C.R. Mahajan, P.A. Patil, S.K. Lad and P.M. Phumal. 1994. Variability, heritability, correlation, path analysis and genetic divergence studies in upland rice. IRRI notes, 19: 8-10.

13. Mesbah, M., H.R. Soroush and A.H.H. Zadeh. 2004. A study of the relationship between grain yield components in rice. Iranian Journal of Agriculture Science, 5: 983-993.

14. Mohadesi, A. 2011. Research project check compatibility and stability of rice lines in regional yield trials. Rice Research Institute of Iran (RRII). Mazandaran Branch, Agricultural research education and extension organization (AREEO), Amol, Iran, (In Persian).

15. Montazeri, R., M. Moradkhani, M. Sam-daliri and A.B. Mosavi. 2017. Correlation between morphological new genotype of rice in the west mazandaran. Journal of Crop Breeding, 9(22): 191199 (In Persian). 
16. Nor-bakhshian, J. and A.M. Rezaei. 1999. Investigation of traits correlations and path analysis of grain yield in rice. Iranian Journal of Crop Sciences, 1(4): 55-65 (In Persian).

17. Prasad, B., A.K. Patwary and P.S. Biswas. 2001. Genetic variability and selection criteria in fine rice (Oryza sativa L.). Pakistan Journal, 4(10): 1188-1190.

18. Rahimi, M. and B. Rabiei. 2009. Estimation of gene action and heritability of important agronomic traits in rice (Oryza sativa L.). Iranian Journal of Crop Sciences, 10(40): 362-376 (In Persian).

19. Rahimi, M., B. Rabiei, M. Ramezani and S. Movafegh. 2010. Evaluation of agronomic traits and determination of variable for improvement rice yield. Journal of Iranian Field Crop Research, 8: 111119 (In Persian).

20. Rahim-soroush, H., M. Mesbah, H. Hossein-zade and R. Bozorgi-pour. 2004. Genotype variation phenotype and cluster analysis and for the quantitative and qualitative traits study of rice. Seed and Plant Improvement Institute, 20: 167-172 (In Persian).

21. Wright, S. 1921. Correlation and causation. Journal of Agricultural Research, 20: 557-585.

22. Yazdi-samadi, B. and S. Abde-mishani. 2011. Advanced plant breeding. Tehran University press, 408 pp (In Persian). 


\title{
Correlation Analysis between Grain Yield and Some Important Traits Related to Rice Lines using Path Analysis
}

\author{
Maryam Saraei $^{1}$, Morteza Sam-daliri ${ }^{2}$, Ali Mohadesi ${ }^{3}$ and Majid Moradkhani ${ }^{4}$ \\ 1 and 2- Graduated M.Sc. Student and Associate Professor, Islamic Azad University, Chalous Branch, Faculty of \\ Agricultural, Agronomy Department, Chalous, Iran \\ 3- Researcher Rice Research Station Tonekabon, Agronomy and Plant Breeding Research Department, Rice Research \\ Institute of Iran (RRII), Mazandaran Branch, Agricultural Research Education and Extension Organization (AREEO), \\ Amol, Iran \\ 4- Graduated M.Sc. Student, Islamic Azad University, Takestan Branch, Faculty of Agricultural Sciences, Agronomy \\ Department, Takestan, Iran (Corresponding author: moradkhani.majid@ hotmail.com) \\ Received: February 20, $2017 \quad$ Accepted: October 2, 2017
}

\begin{abstract}
The aim of present study was to determine the relationship between grain yield and important agricultural characteristics for six lines of rice and Shiroodi cultivar as control in rice research station (Tonekabon) in west of Mazandaran Province in 2015. The experiment was conducted in a randomized complete block design with three replicates. Analysis of variance showed that there was a significant difference between genotypes for all studied traits except flag leaf width, which indicates the appropriate variation among the studied genotypes. Results of mean comparison test showed that the highest and the lowest grain yield were belong to line No. 5 (7831.7 kg.ha' ${ }^{-1}$ ) and No. 204 (6884.3 kg.ha' $\left.{ }^{-1}\right)$ respectively. Results showed that there were positive and significant correlation between grain yield and number of tiller, 1000-grain weight, filled grain number and number of total grains per panicle. Using stepwise regression method showed that 1000-grain weight, filled grain number and number a tillers had the maximum effect on grain yield. Results of path analysis showed that 1000-grain weight an important and effective trait can be used for selection of high yielding rice lines in breeding programs.
\end{abstract}

Keywords: Rice varieties, Stepwise regression method, Path analysis, Correlation analysis, Yield comparison 